Revue d'histoire de l'Amérique française

DE REVUE D.HISTOIRE DE L'AMÉRIQUE FRANÇAISE

\title{
La Société littéraire et historique de Québec (The Literary and Historical Society of Quebec) 1824-1890
}

\section{Ginette Bernatchez}

Volume 35, numéro 2, septembre 1981

URI : https://id.erudit.org/iderudit/303949ar

DOI : https://doi.org/10.7202/303949ar

Aller au sommaire du numéro

Éditeur(s)

Institut d'histoire de l'Amérique française

ISSN

0035-2357 (imprimé)

1492-1383 (numérique)

Découvrir la revue

Citer cet article

Bernatchez, G. (1981). La Société littéraire et historique de Québec (The Literary and Historical Society of Quebec) 1824-1890. Revue d'histoire de l'Amérique française, 35(2), 179-192. https://doi.org/10.7202/303949ar d'utilisation que vous pouvez consulter en ligne. 


\section{LA SOCIÉTÉ LITTÉRAIRE ET HISTORIQUE DE QUÉBEC (THE LITERARY AND HISTORICAL SOCIETY OF QUEBEC) 1824-1890*}

GINETTE BERNATCHEZ

Département d'histoire

Université Laval

L'étude de la Literary and Historical Society of Quebec présente un intérêt particulier du fait qu'elle fut l'une des premières sociétés savantes à exercer son activité d'une façon permanente à Québec ${ }^{1}$. Elle fut fondée en 1824, conjointement par des Anglais et des Canadiens, et son apport à la vie culturelle de Québec est indéniable.

Nous avons voulu cerner les principaux aspects culturels de cette association en dépouillant et en analysant ses Transactions ${ }^{2}$. Cette approche nous fut en quelque sorte imposée par des contingences extérieures, le fonds de la Literary and Historical Society of Quebec ayant été dispersé au cours des dernières années. Les Transactions constituent toutefois des documents très denses, de première importance en ce qui a trait à l'histoire culturelle et sociale de Québec au XIX ${ }^{\text {ieme }}$ siècle $^{3}$.

La Société existe toujours. Elle loge dans l'édifice du Morrin College, rue Saint-Stanislas, à Québec. Notre étude s'arrête toutefois en 1890. Au départ, nous avions l'intention de clore notre travail à l'année 1870, mais en raison de la dispersion des sources, de l'intérêt qu'elles prenaient à partir de 1870 et du tournant que la

- * Texte d'une communication présentée au Congrès de l'IHAF de 1980. Les données nécessaires à la rédaction de ce texte ont été puisées dans la thèse de maîtrise de l'auteur.

En 1809, la Société littéraire de Québec avait vu le jour sous les auspices de François Romain. Son existence fut cependant très brève.

2 Publications de conférences données devant les membres de la Société et de différentes informations sur la Société: rapports du conseil, du trésorier, du bibliothécaire, du responsable du musée, listes de membres.

3 Malheureusement, entre 1837 et 1860 , en raison de plusieurs problèmes internes, la Société ne publia aucun volume. Nous avons suppléé à cette lacune en dépouillant quelques journaux, soit la Gazette de Québec et le Quebec Mercury. 
Société semblait amorcer à la fin des années 1880 , nous avons prolongé cette étude jusqu'en 1890 .

Notre recherche s'articule en deux parties principales: le recrutement des membres et les principales activités culturelles offertes par la Société, c'est-à-dire les conférences et les publications, la bibliothèque et le musée. Auparavant, il convient toutefois d'esquisser un rappel des grandes dates qui jalonnent l'histoire de cette association.

\section{Rappel historique}

La Literary and Historical Society of Quebec (LHSQ) ${ }^{4}$ vit le jour le 6 janvier 1824, à l'instigation du Comte Dalhousie, gouverneur du Canada. Dès son arrivée au pays, l'établissement d'une société historique fut l'une de ses préoccupations. Nulle société savante n'existait alors à Québec, et pourtant le développement de la ville s'accélérait.

Le 15 mars 1824, une assemblée générale tenue au Château Saint-Louis approuvait les règlements de la nouvelle Société et exposait les projets de cette dernière:

Les premiers et principaux objets de la Société seront donc naturellement de découvrir et de soustraire à la main destructive [sic] du temps les fastes qui peuvent encore exister de l'histoire des premiers temps du Canada, de préserver, tandis que c'est encore en notre pouvoir, tous les documents qui peuvent se trouver dans la poussière de dépôts qui n'ont pas

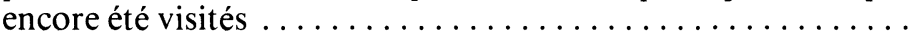

Les objets qui paraissent devoir ensuite attirer l'attention de la Société sont d'encourager par tous les moyens possibles la découverte, la collection et l'acquisition de toutes les informations tendant à répandre du jour sur l'histoire naturelle, civile et littéraire de l'origine des Provinces Britanniques dans l'Amérique Septentrionale... ${ }^{5}$

Lord Dalhousie encouragea d'une façon encore plus tangible les premiers pas de la Société en lui accordant une allocation annuelle de $£ 100$. Son successeur, le gouverneur Sir James Kempt, contribua lui aussi financièrement au succès de l'association et, par la suite, des sommes furent votées régulièrement par les différents gouvernements qui se succédèrent.

$4 \quad$ Nous allons parfois employer le diminutif $L H S Q$. Cette expression était utilisée régulièrement par les membres de la Literary and Historical Society of Quebec pour désigner leur Société.

5 Anonyme. Quebec Literary and Historical Society cité dans Louis-P. Turcotte, «Histoire de la Société Littéraire et Historique depuis sa fondation», Transactions of the Literary and Historical Society of Quebec, New Series, XIII (Sessions of 1877-8-9): 25. 
En 1827, une société similaire vit le jour à Québec sous la présidence du colonel Joseph Bouchette. Elle prit le nom de Société pour l'Encouragement des Sciences et des Arts au Canada. Cette Société put rapidement se glorifier de la longue liste de ses membres, majoritairement d'origine canadienne-française, contrairement à ceux de la LHSQ. Néanmoins, les membres les plus actifs de l'une se retrouvaient souvent à la tête de l'autre.

En 1829, Sir James Kempt, patron de la nouvelle Société, comme il le fut de la première, intervint en faveur de leur fusionnement. À cette époque, une seule société offrait peut-être plus de chances de succès que deux associations poursuivant des buts similaires. On ne peut toutefois écarter l'hypothèse que Sir James Kempt, poursuivant en cela les buts de la politique coloniale anglaise, ait voulu réunir dans un même organisme culturel des gens des deux ethnies, en espérant leur inculquer de cette façon une mentalité commune ${ }^{6}$.

Un fait demeure: après la fusion des deux associations, les Canadiens français participèrent relativement peu à l'activité de la nouvelle Société. Après quelque temps, on décida de garder le nom de la plus ancienne des deux sociétés fusionnées, soit la Literary and Historical Society of Quebec/Société littéraire et historique de Québec.

Pendant les années qui suivirent, la Société ne cessa point de prospérer. En 1829, elle publia son premier volume de Transactions. Les membres, qui étaient à ce moment au nombre de 130 , déployaient un zèle constant. Vers 1830 , la bibliothèque et le musée virent le jour grâce à l'augmentation simultanée du nombre de ses membres et des fonds.

La Société prit un tel essor qu'elle demanda une charte royale qui lui fut accordée le 5 octobre 1831 par Guillaume IV, roi d'Angleterre.

La Société n'oubliait pas les buts qu'elle s'était fixés à l'origine. En 1832, elle fit acheter à Paris et à Londres des collections d'ouvrages et de cartes sur l'Amérique. Elle tenta aussi de se procurer des manuscrits concernant notre histoire, mais toutes les tentatives faites en ce sens ne furent pas couronnées de succès.

Ce n'est qu'en 1838 qu'un premier volume intitulé Mémoire sur le Canada depuis 1749 jusqu'à $1760^{7}$ put être mis sous presse.

6 M. Antonio Drolet a déjà émis cette hypothèse dans son volume intitulé Les bibibliothèques canadiennes. 1604-1960 (Montréal, Cercle du livre de France, /1965/), 115. La rédaction de ce texte fut attribuée à Louis-Léonard de France Aumanon, sieur de Courville, par Aegidius Fauteux, "Le S... de C... enfin démasqué», Les Cahiers des Dix (Montréal, numéro Cinq, 1940): 236. 
La Société ne réussit à publier régulièrement des documents historiques qu'à partir de 1860 . Entre-temps, malgré les projets qu'elle continuait d'entretenir, les progrès n'étaient pas toujours évidents.

Les problèmes que la Société devait affronter n'étaient pas tous du même ordre. Les graves questions politiques auxquelles le Bas-Canada dut faire face en 1837-1838 détournèrent les gens des préoccupations culturelles. Qui plus est, le gouvernement du BasCanada installé à Québec fut aboli et remplacé par le Conseil spécial. En 1841, les parlementaires du Canada-Uni quittèrent Québec pour aller s'installer à Kingston, d'abord, et ensuite à Montréal en 1844.

Pour la Société, cela signifiait la perte de plusieurs membres très actifs et un manque de revenus considérable. À cette époque d'ailleurs, les différents déménagements du siège de la capitale politique ralentirent ses activités durant près de vingt ans. Par la suite, la création de quelques autres sociétés savantes, comme la Quebec Library Association (1843) et l'Institut canadien de Québec (1848), divisèrent «talents et ressources».

Le problème de la recherche de locaux adéquats mina à son tour la santé déjà précaire de la Société durant ces décennies. Pendant les premières années de son existence, elle tint ses réunions et présenta ses conférences au Château Saint-Louis, grâce à la collaboration du gouverneur. Toutefois, après l'incendie du Château en 1834, le gouvernement favorisa l'installation de la bibliothèque de la Société à l'Hôtel Union et, par la suite, dans les locaux du gouvernement. La recherche d'un local constitua un des problèmes majeurs de la Société jusqu'en 1862. À cette date, le Morrin College, qui venait d'ouvrir ses portes, lui céda quelques salles moyennant l'accès à la bibliothèque et au musée pour le personnel de l'institution et ses élèves. Lorsque le Morrin College acheta l'ancienne prison située au coin des rues Sainte-Anne et SaintStanislas, en 1866, il offrit à la Société des locaux décrits comme étant fort spacieux.

Finalement, on ne peut passer sous silence les incendies de 1854 et de 1862 qui éprouvèrent fortement la Société, lui faisant perdre à chaque fois une grande partie de sa bibliothèque et du contenu de son musée. Disons encore qu'il ne fut guère facile de recueillir des renseignements sur la période de 1836 à 1862 environ, période que l'on peut qualifier de peu féconde.

Mais, en 1862, l'entente conclue avec le Morrin College insuffla une nouvelle ardeur à la Société. Elle recommença la publication régulière des Transactions, dont vingt volumes parurent entre cette date et 1890 . Des documents historiques furent également 
publiés régulièrement et la bibliothèque qui ne contenait que 700 volumes environ après l'incendie de 1862 en dénombrait déjà 8500 en 1866 et ce grâce aux dons et aux achats, mais surtout grâce à l'acquisition de la bibliothèque de la Quebec Library Association au cours de cette dernière année.

Les problèmes internes réapparaîtront en 1883, lorsque le gouvernement provincial réduira sa contribution financière de $25 \%$. De 1884 à 1888 , aucune somme ne fut versée à l'institution, ce qui compromit sérieusement son existence. En 1889, grâce à la reprise des subventions gouvernementales, la Société put renouer avec ses activités habituelles mais, déjà, elle avait amorcé une réorientation de ses centres d'intérêt. En 1891, une grande partie du musée fut vendue au High School de Québec et, en 1892, elle abandonna la publication des Transactions pour se consacrer davantage à la recherche historique et à sa bibliothèque.

\section{Le recrutement des membres}

Le problème du recrutement fut abordé bien avant la formation de l'association. En 1823, Lord Dalhousie écrivait à l'Honorable William Smith:

My object would be to form a Society limited in numbers - I would say 24 , but holding in view to augment, as well as to enlarge in scope, as we shall fell our strength and means to do so. ${ }^{8}$

À la réunion du 16 mars 1824 , on adopta le règlement numéro VI qui stipulait qu'une somme de cinq livres serait payable à l'admission et que la souscription annuelle des membres s'élèverait à trois livres. Ce montant était élevé.

Cette situation permit au révérend James Douglas d'écrire que c'est le caractère exclusif de la Société ainsi que le coût élevé de la cotisation annuelle qui entraînèrent la création d'autres sociétés savantes ${ }^{9}$. Comme nous l'écrivions auparavant, la Société pour l'Encouragement des Sciences et des Arts au Canada avait reçu l'appui de plusieurs Canadiens français. C'est ce qui la distinguait d'abord de la Société, mais il faut aussi mentionner le fait qu'elle s'était donné une réglementation beaucoup plus libérale.

En 1831, quelques années à peine après la fusion, on estime à $23 \%$ le nombre de Canadiens français au sein de la Société. C'est d'ailleurs à partir de cette époque que celle-ci s'est démocratisée en

14.

8 Dr. Henry Ievers, «History of the Society», Transactions of the L.H.S.Q., 30:

9 Reverend James Douglas, "Opening Address», Transactions of the L.H.S.Q., New Series, Part 4 (Session of 1865-6): 9. 
abaissant tout au moins le montant de la cotisation exigée. Le sociétaire devait toujours payer cinq livres à l'admission, mais seulement une livre et cinq shillings par année, par la suite. Le fusionnement signifiait donc, pour le moment, un accroissement des membres et, de ce fait, une augmentation des revenus.

La Société admit alors dans ses rangs un plus grand nombre de membres associés. Le conseil d'administration se composait d'une douzaine de personnes et le nombre des membres correspondants oscilla entre 30 et 50 sociétaires. Elle recevait régulièrement des lettres des quatre coins du monde et davantage des quatre coins du Québec. Elle fut bien représentée aux États-Unis, en Angleterre et en France, mais aussi en Espagne et au Danemark pendant plusieurs années. Aux membres correspondants venaient se joindre les membres honoraires, dont le nombre était limité à 20 , et à partir de 1866 , les membres privilégiés qui étaient en réalité les anciens sociétaires de la Quebec Library Association.

Entre 1829 et 1890 , les différentes listes de membres publiées dans les Transactions nous apprennent que le nombre de membres varia entre 116 (minimum enregistré en 1865) et $372(1883)$, pour la période étudiée. Par contre, certains rapports du conseil nous révèlent qu'en 1850, par exemple, 14 personnes seulement avaient payé leur cotisation ${ }^{10}$.

Quant à la participation numérique des Canadiens français, elle se situa à environ $17 \%$ des membres, de 1876 à 1890 . Elle avait atteint son sommet en 1866 avec $33 \%$. Faut-il penser que certaines personnes s'enthousiasmaient alors pour le projet confédératif imminent et auraient ainsi manifesté leur désir de collaborer davantage avec le groupe anglophone? Quoi qu'il en soit, ce regain de coopération fut de courte durée.

Il ne faudrait cependant pas se hâter de conclure à la non participation canadienne-française aux activités intellectuelles de l'époque. Il serait plus juste de dire que les francophones se trouvaient dans une position plutôt inconfortable au sein d'une société savante majoritairement anglophone, même si cette dernière se voulait représentante des deux groupes ethniques à Québec. Dans les faits, la Literary and Historical Society of Quebec - Société littéraire et historique de Québec n'était bilingue que de nom.

Certains membres canadiens-français influents de la Société s'expliquaient mal l'indifférence de leurs compatriotes à leur égard, mais ces derniers, pour des raisons sociales et politiques pouvaient fort bien désirer des institutions autonomes. La Société 
fit assez peu pour attirer l'élément francophone de la population. Les réunions se déroulaient en anglais, et cette langue était employée pour les publications régulières et les conférences. Il faut dire que le premier document historique imprimé était de langue française; le mérite en revient d'ailleurs à Georges-Barthélémi Faribault. De fait, certains Canadiens français jouèrent un rôle important, tels que Pierre-Joseph-Olivier Chauveau, Faucher de Saint-Maurice et James McPherson LeMoine, mais ces figures de proue ne réussirent pas vraiment à entraîner leurs compatriotes. Ajoutons, à titre indicatif, que les Canadiens français occupèrent environ $14 \%$ des sièges au conseil d'administration, pourcentage inférieur à celui de leur participation numérique réelle.

Le conseil était surtout composé de membres du parlement, d'officiers de la garnison, de membres des professions libérales ainsi que de quelques scientifiques. Ces derniers faisaient généralement partie de l'armée ou de la marine. Il faut, par exemple, retenir le nom du capitaine Frederick Henry Baddeley qui, entre les années 1829 et 1835 surtout, présenta devant la Société des travaux de géologie d'une grande valeur.

Le premier conseil se composait du président, le lieutenantgouverneur Francis E. Burton, des vice-présidents, les juges Sewell et Vallières de Saint-Réal, du secrétaire, William Green, savant distingué, et du trésorier, John Charlton Fisher, imprimeur de la Reine. Les hommes qui les suivirent se distinguèrent tous, tant par leur science que par leur situation.

Il convient de signaler que la plupart des membres du conseil ne se contentaient pas d'administrer la Société. Ils étaient au coeur de toutes les activités. L'apport du président, du bibliothécaire et du conservateur du musée s'évalue difficilement, mais il est indéniable que des gens tels John Langton, le révérend James Douglas et James McPherson LeMoine firent énormément pour l'association. Ce sont eux finalement qui permirent à la Société de s'adapter et de traverser les années plus difficiles.

Par ailleurs, la mobilité de ses membres fut relativement faible. Le fait de retrouver les mêmes noms d'une année ou même d'une décennie à l'autre laisse entendre que la Société éprouvait des difficultés à rajeunir son administration. Selon le révérend James Douglas, peu de jeunes gens s'intéressaient à l'association ${ }^{11}$. Il faut peut-être voir là une autre des raisons qui expliqueraient la désertion de la Société à une certaine époque. Il semble que celle-ci ait eu en réalité beaucoup de difficultés à se départir de son caractère élitiste et ce, peut-être même malgré elle.

11 Douglas, loc. cit., 14. 


\section{Les activités culturelles}

C'est à partir d'une étude des conférences présentées, des documents publiés, de la bibliothèque et du musée, que nous avons tenté de dégager les centres d'intérêt privilégiés par la Société. L'objet de notre analyse était en fait de rechercher l'orientation véritable de ces différentes activités, car celle-ci fut parfois accusée d'opérer une sorte de divorce entre les buts fixés au départ et son activité réelle.

\section{a) Conférences et publications}

Les conférences constituaient une des préoccupations majeures de l'organisme. En 1891, le bibliothécaire Frederick C. Würtele publia un index de toutes les conférences présentées devant la Société entre 1829 et $1891^{12}$. Ce document nous apprend que 374 conférences furent prononcées durant cette période et, de ce nombre, 190 furent publiées pour former les Transactions.

Un des faits les plus intéressants à noter concerne les auteurs de ces conférences. En effet, près de la moitié d'entre eux firent partie du conseil d'administration. Généralement, ces «lectures» publiques ne s'adressaient qu'aux membres et à leurs invités. Elles n'en demeuraient pas moins à l'époque une activité sociale de choix, même si les sujets dont on s'entretenait peuvent nous sembler quelque peu rébarbatifs.

En 1862, le chimiste Thomas-Sterry Hunt, personnage bien connu et estimé à cette époque, attira plus de 500 personnes à l'une de ses conférences. Il était suivi ce soir-là du révérend James Douglas qui entretint son auditoire de la religion chez les anciens Égyptiens ${ }^{13}$. L'analyse des principaux sujets traités dans le cadre de ces «lectures» publiques souligne l'importance des sciences naturelles qui occupent la première place avec 70 conférences, suivies de l'histoire profane (66), des sciences physiques et mathématiques (51) et de la géographie (46). Malgré des efforts de vulgarisation évidents de la part des conférenciers, ces causeries ne devaient souvent s'adresser qu'à un public averti. Néanmoins, d'après les rapports du conseil, elles attirèrent toujours plusieurs personnes, tout au moins durant les années où la Société littéraire et historique de Québec n'était pas aux prises avec des problèmes internes.

12 Ce document fut publié à nouveau en 1927. La référence exacte de ce document est: The Literary and Historical Society of Quebec. Index of the Lectures, Papers and Historical Documents published by the Literary and Historical Society of Quebec, and also of the Names of their Authors, together with a List of Unpublished Papers read before the Society, 1829 to 1891, Extended to 1927 (inclusive). (Québec, 1927). 49 pages.

i3 Gazette de Québec, 16 mai 1862. 
Plusieurs de ces conférences, comme nous l'avons mentionné, firent l'objet de publications, mais la Société publia aussi de nombreux documents historiques. C'était là d'ailleurs le but principal de l'association et il est vrai que celle-ci a tardé à remplir cet objectif, puisque le premier document historique imprimé ne fut mis sous presse qu'en 1838. Assez curieusement, cela coïncide avec un déclin de l'activité scientifique de la Société ${ }^{14}$. Celle-ci s'intéressait toutefois depuis fort longtemps à la conservation et à la publication d'archives. C'était une tâche ardue, car les moyens financiers faisaient défaut. Elle n'obtint des fonds pour cette initiative qu'en 1832.

La $L H S Q$ réussit à publier plusieurs volumes de documents historiques, même si les premières tentatives entreprises en ce sens ne furent pas très heureuses. Les moyens dont l'association disposa au début de sa création ne lui permirent donc pas de réaliser immédiatement ses objectifs. De plus, il était bien tentant de profiter de ce que l'historien Richard Jarrell a appelé «l'âge d'or» des sciences à Québec ${ }^{15}$. La $L H S Q$ fit d'ailleurs plus que profiter de ce courant scientifique, elle y contribua largement en présentant plusieurs travaux d'envergure, notamment dans les domaines de la géologie, de la minéralogie et des sciences biologiques. On ne peut passer sous silence les noms du capitaine $\mathrm{H}$. W. Bayfield, du docteur William Kelly et de l'Honorable William Sheppard of Woodfield.

Les administrateurs de la Société se sentaient quelque peu fautifs de privilégier le domaine des sciences naturelles au détriment de la recherche historique. Ils ne manquaient jamais de faire état dans leurs rapports des efforts accomplis afin de faire progresser leurs recherches en histoire. Néanmoins, la Société a joué un rôle de premier plan en ce qui concerne la conservation et la publication d'archives et ce, à une époque où ce secteur culturel était somme toute mal organisé.

En 1888, le gouvernement du Québec réquisitionna une série fort imposante de documents manuscrits qui étaient entre les mains de la Société. Celle-ci remit à ce moment-là au gouvernement dix volumes de manuscrits originaux de langue française datant des XVII ${ }^{\text {ieme }}$ et XVIII ${ }^{\text {ieme }}$ siècles, et trente et un volumes de manuscrits recopiés portant aussi sur l'histoire du Canada.

\footnotetext{
14 L'historien Richard Jarrell considère que ce déclin se situe entre les années 1837 et 1844, dans son article intitulé «The Rise and Decline of Science at Quebec, 1824-1844», Histoire sociale/Social History, IX, no 19 (mai 1977): 77-91.

15 Ibid.: 83.
} 
La Société conserva d'autres documents de moindre importance qui étaient tout de même d'un grand intérêt historique. Pendant plusieurs années, elle joua, pour ainsi dire, le rôle que devaient tenir plus tard les Archives canadiennes et provinciales. Elle se consacra d'ailleurs davantage à cette activité en 1892 lorsqu'elle abandonna la publication de ses Transactions ${ }^{16}$.

\section{b) Bibliothèque}

La bibliothèque de la Société vit le jour vers 1830 . Un catalogue succinct publié en 1831 nous permet d'évaluer son contenu à 152 volumes $^{17}$. Les rayons se garnirent graduellement grâce à des achats importants, mais surtout grâce aux dons qui constituèrent une source d'approvisionnement non négligeable, surtout durant les années de formation.

Plusieurs volumes de sciences naturelles, notamment de minéralogie, ainsi que plusieurs recueils d'histoire moderne enrichirent son contenu. Ainsi, dès le début, elle prenait la même orientation que les conférences.

En 1845, un premier catalogue plus détaillé fut imprimé, mais le piètre état de la situation financière avait déjà obligé le bibliothécaire à annuler plusieurs abonnements à divers journaux et revues scientifiques. Seulement 26 membres se prévalurent cette année-là de leur privilège de lecteur, ils empruntèrent 222 volumes ${ }^{18}$.

Les incendies de 1853 et de 1862 ébranlèrent fortement les assises de la Société en détruisant notamment plusieurs documents manuscrits pratiquement irremplaçables. Il fallut, pour ainsi dire, tout recommencer. Mais, comme il arrive souvent, ces conflagrations éveillèrent la sympathie des gens et les dons se firent nombreux. Des subventions gouvernementales, jointes au montant des assurances, permirent à l'administration de rendre la bibliothèque de nouveau accessible à ses membres.

En 1864, un autre catalogue fut publié: 1525 titres y figurent, pour un total de 3381 volumes ${ }^{19}$. Cette fois-ci, ce sont les volumes d'histoire moderne qui sont les plus nombreux, suivis des ouvrages de sciences naturelles et de géographie. En fait, il eût été plus juste de baptiser la LHSQ: "Société historique et scientifique de Québec». La littérature n'y était pas, du moins encore en 1864, l'une

\footnotetext{
16 Un dernier volume de Transactions parut cependant en 1924 pour commémorer le centenaire de la Société.

17 L.H.S.Q., Transactions of the Literary and Historical Society of Quebec, II (1831): 439-443.

18 Gazette de Québec, 13 janvier 1845, 3.

19 L.H.S.Q., Catalogue of Books in the Library of the Literary and Historical Society of Quebec (Québec, 1864). 114 pages + Index.
} 
des branches du savoir les mieux représentées. La bibliothèque possédait plusieurs recueils de poésie anglaise et plusieurs volumes de base en histoire littéraire, mais les ouvrages d'art dramatique et les romans de littérature populaire étaient presque absents des rayons. Au premier abord, cela peut étonner puisque le roman, par exemple, prenait à cette époque un essor qu'il n'a jamais perdu depuis.

Toutefois, la bibliothèque n'était pas ce que l'on appelle communément une bibliothèque générale. Selon les désirs de ses fondateurs, elle devait répondre à des objectifs bien précis, soit l'avancement de l'histoire, des sciences et, dans une certaine mesure, de la littérature. En somme, la même ligne de pensée guidait les administrateurs dans le choix des conférences à présenter, des volumes à acheter et des publications à offrir.

Les auteurs français étaient peu nombreux sur les rayons (environ 7\%), mais tous les grands classiques y trouvaient leur place, de Rabelais à Jean-Jacques Rousseau, en passant par Molière et Lafontaine. Certains d'entre eux étaient d'ailleurs traduits en anglais, tels Jean Froissart et Auguste Comte. La bibliothèque possédait en outre plusieurs ouvrages des grands écrivains français du XIX ${ }^{\text {ieme }}$ siècle, tels Jules Michelet, François Guizot et Victor Cousin.

La plupart des volumes sur les rayons étaient cependant d'auteurs britanniques, en raison des antécédents culturels de la majorité des sociétaires et, il va sans dire, parce que la tradition littéraire, historique et scientifique était plus ancienne en GrandeBretagne qu'en Amérique anglo-saxonne. On y trouvait, par exemple, les oeuvres de Charles Lamb, Thomas Babington Macaulay, John Stuart Mill et William Prout, pour ne nommer que ceuxlà.

Un troisième catalogue fut imprimé en 1873. La Literary and Historical Society of Quebec avait fait l'acquisition, sept ans auparavant, de la bibliothèque de la Quebec Library Association, au coût de $\$ 500$. L'achat de ces volumes enrichit considérablement les sections de la littérature et de l'histoire modernes, et toutes les branches de ce que l'on appelle aujourd'hui les sciences humaines augmentèrent de plusieurs titres.

Par contre, les volumes de sciences naturelles et de physique ne s'accrurent pas dans une même proportion. D'ailleurs, c'était une façon pour les administrateurs de répondre à la demande de leurs membres. Le conseil en était venu à devoir choisir entre l'achat d'ouvrages scientifiques et de volumes de littérature plus en demande. 
Vers 1883 , le nombre de volumes en circulation s'élevait à 3000 par année. Malheureusement, les problèmes financiers qui réapparurent à cette époque forcèrent l'institution à abandonner plusieurs abonnements à divers journaux et magazines. D'autre part, l'achat de nouveaux volumes se raréfia. La bibliothèque n'en demeurait pas moins une des activités les plus dynamiques de l'association. En 1887, elle occupait la quatorzième place en importance sur la liste des bibliothèques publiques au Canada ${ }^{20}$. C'était une place fort enviable puisqu'on évaluait à 165 le nombre des bibliothèques publiques au Canada à ce moment-là. D'ailleurs, aujourd'hui encore, cette bibliothèque occupe quelques salles du Morrin College et constitue une des activités principales de la Société.

\section{c) Musée}

Le musée fut créé à la même époque que la bibliothèque (vers 1830). Plusieurs pièces de collection avaient été recueillies auparavant mais les administrateurs profitèrent de l'allocation de $£ 250$ donnée par le gouvernement du Bas-Canada en 1830 pour se procurer des instruments et du matériel scientifique.

Au début de sa création, il faut en convenir, le musée se tailla une place fort importante dans la Société. L'histoire naturelle, comme nous l'avons dit, occupait une situation de choix et, de 1829 à 1839 , les dons faits au musée dépassèrent largement ceux qui furent accordés à la bibliothèque. La nature de ces dons était variée, mais on fit une large place à la zoologie et à la minéralogie.

Le musée profita sans doute aussi beaucoup du prestige de la classe d'histoire naturelle, dont le docteur Joseph Skey était titulaire en 1830. Celui-ci fit circuler à travers la province «une adresse» pour attirer l'attention de la population sur l'importance de l'histoire naturelle. Il invita les gens à communiquer à la Société tout renseignement ou spécimen susceptible d'enrichir un sujet $d^{\prime}$ histoire naturelle ${ }^{21}$. Son appel ne demeura pas vain puisque plusieurs y répondirent.

Le musée suivit cependant une courbe bien différente de celle de la bibliothèque. Encore une fois, devons-nous y voir un autre signe du déclin de l'intérêt envers les sciences à Québec? C'est possible. Avec les années, il devint peu à peu le parent pauvre de l'institution. Ce n'était pourtant pas les collections qui manquaient,

20 James Bain jr., cité dans F. C. Würtele, "Our Library», Transactions of the Literary and Historical Society of Quebec, no 19 (Sessions 1887 to 1889): 69.

21 Joseph Skey, "An Address by Joseph Skey, Esq. M. D.», Transactions of the Literary and Historical Society of Quebec, First Series, II (1831): 445. 
mais la Société ne réussissait pas toujours à mettre en valeur tout le matériel qu'elle possédait.

En 1870, James McPherson LeMoine devint conservateur du musée. Il occupa ce poste pendant huit ans et il fut sans contredit celui qui y consacra le plus d'énergie. Selon lui, ce qui avait guidé la Société dans la formation du musée était d'abord le désir de se procurer un spécimen de chaque espèce de la faune canadienne et, par la suite, de la faune américaine et européenne. Mais le musée possédait aussi d'autres pièces importantes dont, entre autres, une collection numismatique de grande valeur.

Le principal problème du conservateur était de composer avec l'exiguité des locaux alloués, qui limitait notamment l'expansion de la collection des grands mammifères canadiens, et les possibilités d'exposer l'ensemble des divers éléments que possédait la Société.

À cela, il faut ajouter les restrictions budgétaires qui empêchèrent sans doute, et à plusieurs reprises, le musée de se développer comme il aurait pu le faire. C'est le domaine de l'ornithologie qui demeura toujours le secteur privilégié, et plus particulièrement celui de l'oologie ${ }^{22}$. Évidemment, cette collection exigeait un espace beaucoup moins vaste que celui des grands mammifères.

De 1884 à 1889, l'appellation «musée» n'apparaît plus dans les rapports du trésorier. Il nous fut même impossible de connaître les montants exacts alloués aux collections. La Société devait faire face à ce moment à des difficultés financières d'envergure, mais il est tout de même permis de s'étonner de ce silence. Finalement, seul ce mutisme laissait présager la décision qu'allait adopter le conseil en 1891 de vendre la plus grande partie du musée, celle concernant les sciences naturelles. C'est le High School de Québec qui en devint l'acquéreur.

Il semble bien que l'espace occupé par le musée ait toujours été trop restreint pour l'ampleur des collections. Mais le musée semblait aussi avoir perdu au fil des ans beaucoup d'intérêt aux yeux des administrateurs et auprès des sociétaires. Une bibliothèque bien garnie répondait sans doute davantage aux besoins immédiats des membres. Les difficultés financières contraignirent donc la Société à faire un choix. 


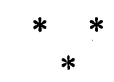

Malgré les nombreux obstacles qu'elle eut à franchir, la Literary and Historical Society of Quebec sut se distinguer pendant tout le XIX ${ }^{\mathrm{e}}$ siècle. Pionnière des sociétés savantes dans les domaines de l'histoire et des sciences, elle ouvrit la voie à plusieurs autres associations. Il aurait sans doute été plus intéressant de situer davantage cette Société dans le contexte des autres sociétés savantes de l'époque, mais la littérature sur le sujet est peu abondante. On ne peut cependant passer sous silence les travaux d'Yvan Lamonde sur le mouvement des associations culturelles. Ce dernier souligne d'ailleurs qu'une étude des sociétés littéraires anglophones du Québec s'impose pour expliquer l'origine des sociétés francophones ${ }^{23}$.

Nous avons voulu avant tout comprendre l'activité intellectuelle des classes supérieures de langue anglaise à Québec au siècle dernier. Les problèmes auxquels la Literary and Historical Society of Quebec était aux prises ne lui étaient cependant pas particuliers. Le dynamisme d'une société ne tient évidemment pas uniquement à des questions monétaires, mais ces questions réflètent souvent le contexte politique d'une époque.

Malgré les lacunes de la documentation disponible, l'étude de cette association nous a permis de répondre à plusieurs interrogations sur le fonctionnement interne d'une société savante en milieu urbain au XIX ${ }^{e}$ siècle. Cette approche éclaire toutefois d'autres aspects fort importants de la question, tel le dynamisme de l'activité scientifique à Québec à une certaine époque.

${ }^{23}$ Voir dans Recherches sociographiques (XVI, no 2, 1975): Yvan Lamonde, «Inventaire des études et des sources pour l'étude des associations «littéraires» québécoises francophones au 19e siècle (1840-1900)», 261-275. À propos de l'idée d'association, voir dans Histoire sociale/Social History (nov. 1975), Yvan Lamonde, «Les associations au Bas-Canada: de nouveaux marchés aux idées (1840-1867)»: 361 à 370. 